\title{
Proteomic Profiling of Bifidobacterium bifidum S17 Cultivated Under In Vitro Conditions
}

OPEN ACCESS

Edited by:

Francesca Turroni,

University College Cork, Ireland

Reviewed by:

Michael C. Nelson University of Connecticut, USA

Marco Ventura,

University of Parma, Italy

Reetta Satokari

University of Helsinki, Finland

${ }^{*}$ Correspondence: Jing Yuan

yuaniing6216@163.com;

Yansong Sun

sunys@qq.com;

Christian U. Riedel

christian.riede/@uni-ulm.de

Specialty section This article was submitted to Microbial Symbioses, a section of the journal

Frontiers in Microbiology

Received: 04 November 2015

Accepted: 18 January 2016

Published: 12 February 2016

Citation:

Wei X, Wang S, Zhao X, Wang X, Li H,

Lin W, Lu J, Zhurina D, Li B, Riedel CU, Sun Y and Yuan J (2016) Proteomic Profiling of Bifidobacterium bifidum $S 17$ Cultivated Under In Vitro

Conditions. Front. Microbiol. 7:97. doi: 10.3389/fmicb.2016.00097

\section{Xiao Wei', Simiao Wang ${ }^{1}$, Xiangna Zhao ${ }^{1}$, Xuesong Wang ${ }^{1}$, Huan Li', Weishi Lin', Jing Lu' ${ }^{1}$, Daria Zhurina ${ }^{2}$, Boxing Li', Christian U. Riedel2*, Yansong Sun ${ }^{1 *}$ and Jing Yuan ${ }^{1 *}$}

${ }^{1}$ Institute of Disease Control and Prevention, Academy of Military Medical Sciences, Beijing, China, ${ }^{2}$ Institute of Microbiology and Biotechnology, University of UIm, Ulm, Germany

Bifidobacteria are frequently used in probiotic food and dairy products. Bifidobacterium bifidum $\mathrm{S} 17$ is a promising probiotic candidate strain that displays strong adhesion to intestinal epithelial cells and elicits potent anti-inflammatory capacity both in vitro and in murine models of colitis. The recently sequenced genome of B. bifidum S17 has a size of about 2.2 Mb and encodes 1,782 predicted protein-coding genes. In the present study, a comprehensive proteomic profiling was carried out to identify and characterize proteins expressed by B. bifidum S17. A total of 1148 proteins entries were identified by liquid chromatography coupled to tandem mass spectrometry (LC-MS/MS), representing $64.4 \%$ of the predicted proteome. 719 proteins could be assigned to functional categories according to cluster of orthologous groups of proteins (COGs). The COG distribution of the detected proteins highly correlates with that of the complete predicted proteome suggesting a good coverage and representation of the genomic content of $B$. bifidum S17 by the proteome. COGs that were highly present in the proteome of B. bifidum S17 were Translation, Amino Acid Transport and Metabolism, and Carbohydrate Transport and Metabolism. Complete sets of enzymes for both the bifidus shunt and the Embden-Meyerh of pathway were identified. Further bioinformatic analysis yielded 28 proteins with a predicted extracellular localization including 14 proteins with an LPXTG-motif for cell wall anchoring and two proteins (elongation factor Tu and enolase) with a potential moonlighting function in adhesion. Amongst the predicted extracellular proteins were five of six pilin proteins encoded in the B. bifidum S17 genome as well as several other proteins with a potential role in interaction with host structures. The presented results are the first compilation of a proteomic reference profile for a B. bifidum strain and will facilitate analysis of the molecular mechanisms of physiology, host-interactions and beneficial effects of a potential probiotic strain.

Keywords: proteomic profiling, metabolic pathways, pilin proteins, LC-MS/MS, Bifidobacterium bifidum

Abbreviations: DTT, DL-dithiothreitol; ECM, extracellular matrix; EDTA, ethylene diamine tetraacetie acid; HEPES, 4-(2hydroxyethyl)-1-piperazineethanesulfonic acid; IECs, intestinal epithelial cells; KEGG, Kyoto Encyclopedia of Genes and Genomes; LC-MS/MS, liquid chromatography coupled to tandem mass spectrometry; LPXTG, Leu-Pro-X-Thr-Gly; MRS, Man-Rogosa-Sharpe; ORF(s), open reading frame(s); PMSF, phenylmethanesulfonyl fluoride; ThDP, thiamine diphosphate; TMDs, transmembrane domains; UHPLC, ultra-high-performance liquid chromatography. 


\section{INTRODUCTION}

Bifidobacteria represent an important group of the human intestinal microbiota (Bottacini et al., 2014). Due to their reported ability to reduce cholesterol levels, exclude intestinal pathogens, strengthen the intestinal barrier, alleviate symptoms of constipation, and/or modulate the immune response they are frequently used as active ingredients in food and dairybased products (Leahy et al., 2005; Gareau et al., 2010). In order to reveal the molecular mechanisms responsible for these beneficial effects, several bifidobacterial strains have recently been sequenced (Ventura et al., 2009). Analysis of bifidobacterial genomes has led to the identification of various structures involved in host colonization and probiotic properties. For example, Bifidobacterium breve UCC2003 was shown to encode genes for production of exopolysaccharides (Fanning et al., 2012). These exopolysaccharides support persistence in the murine gastrointestinal tract and are required for the protective effect of $B$. breve UCC2003 against infections with the murine pathogen Citrobacter rodentium. B. breve UCC2003 also possesses type IV tight adherence pili and these pili were shown to support long term colonization of mice (O'Connell Motherway et al., 2011). Similarly, other strains and species of bifidobacteria were shown to contain gene clusters for Tad and/or sortase-dependent pili for some of which interaction with host structures has been shown (Foroni et al., 2011; Turroni et al., 2013, 2014b).

Bifidobacterium bifidum strains belong to the infant-type bifidobacteria and show a remarkable adaptation to their ecological niche in the intestinal tract of human neonates. This includes a large number of adhesive structures and a specific ability to utilize host-derived glycans (Turroni et al., 2014a). B. bifidum S17 was isolated from feces of a breast-fed infant and displays unusually strong adhesion to IECs (Riedel et al., 2006a; Preising et al., 2010; Gleinser et al., 2012). Additionally, the strain elicits a promising anti-inflammatory capacity both in vitro (Riedel et al., 2006b; Preising et al., 2010) and in vivo in three different murine models of colitis (Preising et al., 2010; Philippe et al., 2011; Grimm et al., 2015). The genome of B. bifidum S17 was sequenced and annotated and contains a predicted 1,782 protein-coding ORFs (Zhurina et al., 2011) including one Tad and three sortase-dependent pili gene clusters as well as several other genes suspected or shown to play a role in adhesion to host structures or host colonization (Gleinser et al., 2012; Westermann et al., 2012).

In recent years, proteomic analysis has become an indispensible tool to analyze the biology of microorganisms, their response to changes in the environmental conditions and their interaction with the host (Otto et al., 2014). One of first the reports on a proteomic analysis of a Bifidobacterium sp. strain was a proteomic reference map obtained by $2 \mathrm{D}$ electrophoresis and MALDI TOF-TOF mass spectrometry (Yuan et al., 2006). Since then, this technique has been used to study adaptation of various bifidobacteria to bile and oxidative stress (Sánchez et al., 2007; Xiao et al., 2011) and different carbon sources (Liu et al., 2011, 2015), host-induced proteome changes of B. longum NCC2705 (Yuan et al., 2008) or its interaction with IECs (Wei et al., 2014). Turroni et al. (2010) performed a proteomic analysis of B. bifidum PRL2010 during growth on different sugars and following contact with cultured epithelial cells (Turroni et al., 2010).

However, proteomic approaches that include 2D electrophoresis have limitations in the detection of alkaline and low-abundance proteins (Otto et al., 2014). These limitations can be overcome by $1 \mathrm{D}$ gel-based LC-MS/MS (Wickramasekara et al., 2011; Otto et al., 2014). Moreover, LC-MS/MS is more efficient and accurate when analyzing differential global protein expression quantitatively and was used for proteomics of Burkholderia vietnamiensis (Wickramasekara et al., 2011). Another example is the analysis of secretion profiles of B. pseudomallei MSHR668 (Burtnick et al., 2014). LC-MS/MS has also been employed for comparative proteomics of two Lactobacillus rhamnosus strains (Savijoki et al., 2011). For bifidobacteria, LC-MS/MS was used for differential proteomics of two B. longum strains (Guillaume et al., 2009) and a proteomic profiling of B. longum subsp. infantis during growth on lactose, glucose, and galacto-, fructo-, and human milk oligosaccharides (Kim et al., 2013).

In the present study, the proteome of B. bifidum S17 grown in vitro was analyzed by $1 \mathrm{D}$ gel-based ultra-high performance LCMS/MS representing the first comprehensive proteomic profile for the species B. bifidum.

\section{MATERIALS AND METHODS}

\section{Bacterial Strains and Growth Conditions}

Bifidobacterium bifidum S17 was cultured in sealed jars anaerobically at $37^{\circ} \mathrm{C}$ in Lactobacillus MRS medium (Difco) supplemented with $0.05 \%$ L-cysteine. Anaerobic conditions were achieved and maintained using AnaeroGen sachets (Thermo Scientific). Bacteria were harvested for proteomic analysis in midexponential growth phase at an optical density at $600 \mathrm{~nm}$ of 0.9 corresponding to approximately $1.5 \times 10^{8}$ colony forming units/ml.

\section{Preparation of Whole Cell Protein Extracts}

Bacteria were harvested by centrifugation, washed twice in phosphate-buffered saline (PBS), and pellets (about $0.30 \mathrm{~g}$ ) were resuspended in $5 \mathrm{~mL}$ of lysis buffer $(8 \mathrm{M}$ urea, $30 \mathrm{mM}$ HEPES, $1 \mathrm{mM}$ phenylmethylsulfonyl fluoride, $2 \mathrm{mM}$ ethylenediaminetetraacetic acid, $10 \mathrm{mM}$ dithiothreitol) containing one dissolved tablet of complete protease inhibitor (Roche Diagnostics, Mannheim, Germany). Bacteria were then sonicated for $10 \mathrm{~min}$ on ice using a Sonifier 750 (Branson Ultrasonics Corp., Danbury, CT, USA) with the following conditions: 2 s of sonication with a 3 s interval set at $25 \%$ duty cycle. The cell lysate suspension was centrifuged for $30 \mathrm{~min}$ at $20,000 \times g$ to collect the supernatant. The proteins were reduced with $10 \mathrm{mM}$ dithiothreitol at $56{ }^{\circ} \mathrm{C}$ for $1 \mathrm{~h}$, and alkylated with $55 \mathrm{mM}$ iodoacetamide at room temperature for $1 \mathrm{~h}$ in the dark. The treated proteins were precipitated in acetone at $-20^{\circ} \mathrm{C}$ for $3 \mathrm{~h}$. After centrifugation at $20,000 \times g$ for $30 \mathrm{~min}$, the protein pellet was resuspended and ultrasonicated in pre-chilled $50 \%$ tetraethylammonium bromide buffer with $0.1 \%$ sodium dodecyl 
sulfate (SDS). The proteins were regained after centrifugation at $20,000 \times g$ and the protein concentrations were measured by Bradford assay.

\section{D SDS Polyacrylamid Gelelectrophersis (SDS-PAGE) and in-Gel Digestion}

The proteome was analyzed by 1D gel-based LC-MS/MS as described previously (Albrethsen et al., 2010). In brief, the proteins were separated via SDS polyacrylamid gelelectrophersis (SDS-PAGE) using precast 4-12\% gradient gels containing BisTris buffer (NuPAGE MES system, Invitrogen). Protein gels were run by using $1 \mathrm{x}$ running buffer [259 $\mathrm{mM}$ Tris base, $2 \mathrm{M}$ glycine, $1 \%(\mathrm{w} / \mathrm{v}) \mathrm{SDS}$ in $\mathrm{ddH}_{2} \mathrm{O}$ ] at a constant voltage of $85 \mathrm{~V}$ for $20 \mathrm{~min}$ followed by $150 \mathrm{~V}$ for $40 \mathrm{~min}$ (until the dye runs off the gel). The gels were then stained with Coomassie R-250. A single lane of stained gel was cut into 10 pieces of approximately the same size and transferred into $1.5 \mathrm{~mL}$ Eppendorf tubes to perform ingel tryptic digestion. Gel bands were destained and tryptically digested as described previously (Yuan et al., 2006). The digested peptides were extracted with $50 \%$ acetonitrile and $2 \%$ formic acid solution. After extraction, the peptides were transferred into $500 \mu \mathrm{L}$ Eppendorf tubes and concentrated using a SpeedVac Concentrator (Savant) and a volume of $10 \mu \mathrm{L}$ containing $15 \mu \mathrm{g}$ of protein was loaded into individual high-performance liquid chromatography (HPLC) autosampler vials and analyzed by liquid chromatography-tandem mass spectrometry (LC$\mathrm{MS} / \mathrm{MS})$.

\section{LC-MS/MS Analysis}

Peptide samples were analyzed by ultra-high performance LC-MS/MS on a quadrupole-Orbitrap mass spectrometer (QExactive; Thermo Fisher, Germany) equipped with $15 \mathrm{~cm}$ (length) by $75 \mu \mathrm{m}$ (inside diameter) column packed with $5 \mu \mathrm{m} \mathrm{C18}$ medium (Thermo Fisher) which was kept at $21^{\circ} \mathrm{C}$ throughout the analysis. Mobile phase A was MilliQ water with $0.1 \%(\mathrm{v} / \mathrm{v})$ formic acid. Mobile phase B was $99.9 \%(\mathrm{v} / \mathrm{v})$ acetonitrile, $0.1 \%$ acetic acid. Gradient was run from $0 \% \mathrm{~B}$ to $30 \% \mathrm{~B}$ over $40 \mathrm{~min}$ and then to $80 \% \mathrm{~B}$ for $15 \mathrm{~min}$. The LC was interfaced to a quadrupole-Orbitrap mass spectrometer (Q-Exactive; Thermo Fisher) via nano-electrospray ionization. An electrospray voltage of $1.8 \mathrm{kV}$ was applied. The mass spectrometer was programmed to acquire, by data-dependent acquisition, tandem mass spectra from the top 20 ions in the full scan from 350 to $2,000 \mathrm{~m} / \mathrm{z}$. Dynamic exclusion was set to $15 \mathrm{~s}$, singly charged ions were excluded, the isolation width was set to $2 \mathrm{~m} / \mathrm{z}$, the full MS resolution was set to 70,000, and the MS/MS resolution was set to 17,500 . Normalized collision energy was set to 28 , automatic gain control to $1 \mathrm{e} 6$, maximum fill MS to $20 \mathrm{~ms}$, maximum fill MS/MS to $60 \mathrm{~ms}$, and the under fill ratio to $0.1 \%$.

\section{Protein Identification and Bioinformatic Analysis}

Peptide identification were performed using Mascot v2.3.01

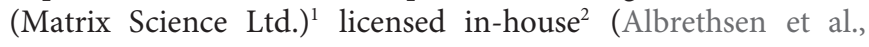

${ }^{1}$ http://www.matrixscience.com

${ }^{2} \mathrm{http} / / / \mathrm{www} \cdot$ proteomics.cn
2010). Peptide mass finger printing searches were performed using Mascot v2.2.06 (Matrix Science Ltd.) ${ }^{1}$ licensed in-house ${ }^{2}$. Monoisotopic peptide masses were used to search the databases, allowing a peptide mass accuracy of $30 \mathrm{ppm}$ and fragment ion tolerance of 0.2 Dalton. Both methionine oxidation and cysteine carboxyamidomethylation were considered in the process. For protein identification by peptide mass fingerprinting, peptide masses were searched against the publically available database for B. bifidum S17 (NCBI Reference Sequence: NC_014616.1). For unambiguous identification of proteins, more than five peptides must be matched and the sequence coverage must be greater than $15 \%$. The complete proteomic dataset is deposited and publically accessible on the iProX database ${ }^{3}$ under project number IPX00067300.

Calculation of protein molecular weights and isoelectric points were carried out by the protparam software from the Expasy toolbox ${ }^{4}$. Functional classification of identified proteins was performed by BLASTPGP (Altschul et al., 1997) searching against the databases of Cluster of Orthologous Groups $\left(\mathrm{COGs}^{5}\right)$ (Tatusov et al., 2000). The cellular localizations of all identified proteins were predicted by PSORTb version 3.0 (Yu et al., 2010). Prediction of signal peptides was carried out with SignalP Version 4.1 (Petersen et al., 2011), TMHMM server 2.0 (Sonnhammer et al., 1998) was used to predict transmembrane helices, and LocateP database (Zhou et al., 2008) was used for prediction of cell wall and lipid anchor motifs.

\section{RESULTS}

The 2.2-Mb genome of B. bifidum S17 contains 1,782 predicted protein-coding ORFs. To obtain an overview on the proteins expressed by $B$. bifidum S17 under standard laboratory conditions, whole protein extracts of bacteria grown in MRS medium were subjected to LC-MS/MS and protein identification. A total of 1,148 proteins were successfully matched unambiguously to one of the 1,782 predicted proteins of B. bifidum S17, i.e., a coverage of $64.4 \%$ of the complete predicted proteome. All detected proteins the peptide data matches the protein sequence as predicted in the genome annotation (data not shown). Moreover, the proteome contained a total of 235 (conserved) hypothetical proteins demonstrating that these proteins are actually expressed by B. bifidum S17 at least under in vitro conditions, which changes their status from "hypothetical" to proteins with unknown function.

Supplementary Table S1 summarizes these proteins along with information on predicted pI, molecular mass, function, signal peptides, and subcellular location. $76.7 \%$ of the B. bifidum S17 proteins detected by LC-MS/MS are acidic with pI between 3 and $7,12.5 \%$ have a pI between 7 and 9, whereas $10.8 \%$ of the proteins have a pI greater than 9 (Figure 1A). Classification of the 1,148 detected proteins into clusters of

\footnotetext{
${ }^{3}$ www.iprox.org

${ }^{4}$ www.expasy.ch/tools/protparam.html

${ }^{5} \mathrm{ftp}$ ://ftp.ncbi.nih.gov/pub/COG/COG2014/static/lists/listCOGs.html
} 
A
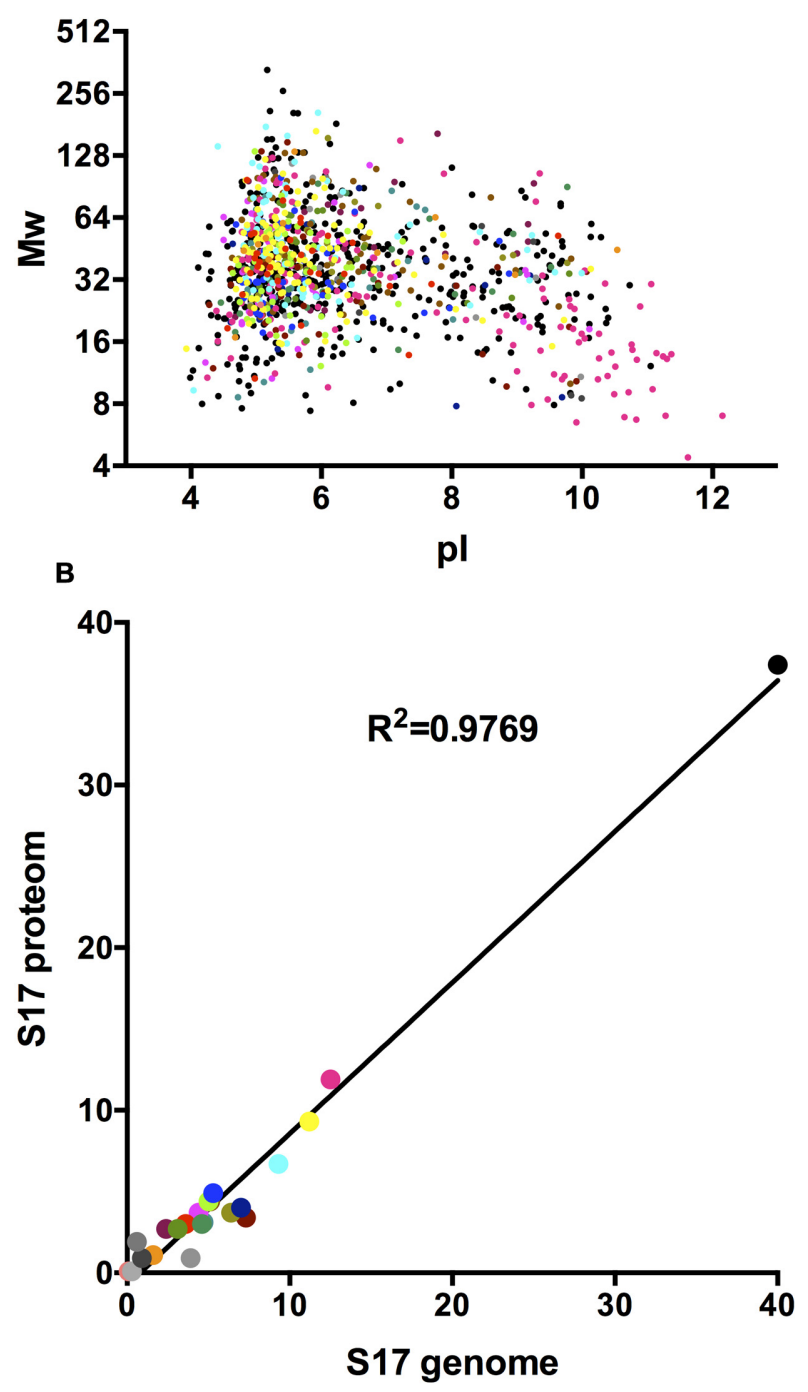

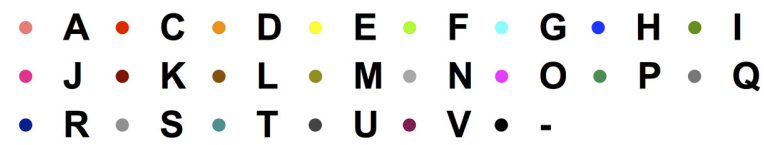

FIGURE 1 | (A) In silico 2D gel electrophoresis of the identified proteins of the B. bifidum S17 proteome. For each identified protein, calculated molecular weight was plotted against the calculated pl. (B) Correlation analysis of the COG distributions of the detected proteins in the proteome and all proteins encoded on the genome of B. bifidum S17.

orthologous groups of proteins (COGs) assigned 719 proteins into 22 functional categories (Table 1). The remaining 429 unclassified proteins $(37,4 \%)$ were denoted as COG-absent proteins. For none of the COGs an obvious clustering according to $\mathrm{pI}$ and $\mathrm{Mw}$ could be observed (Figure 1A). Comparison of the proteomes and genomes of B. bifidum S17 that the COG distribution of the B. bifidum $\mathrm{S} 17$ proteome is highly correlated to the distribution of all 1,782 predicted protein-coding ORFs of the genome (Figure 1B; coefficient of determination $\left.R^{2}=0.9769\right)$. Also, none of the COGs is skewed markedly off the regression in the proteome/genome comparison. Thus, none of the COGs seems to be over- or underrepresented in the proteome compared to the genome. Collectively, this indicates that the proteome captured by LCMS/MS is comprehensive and covers all functional categories to a similar extend as encoded by the genomic information of B. bifidum S17.

The functional categories with highest representation amongst the detected proteins were Translation (functional category J; 137 proteins, $11.9 \%$ of all detected proteins), Amino acid Transport and Metabolism (functional category E; 107 proteins, 9.3\%), and Carbohydrate Transport and Metabolism (functional category G; 77 proteins, 6.7\%). Collectively, $26 \%$ of all detected proteins of the B. bifidum S17 proteome are assigned to functional categories J, A, K, and L. Thus, proteins possessing biological functions related to information storage, DNA replication, recombination and repair, RNA processing, transcription, and translation were highly prevalent in the proteome of B. bifidum S17. Of note, elongation factors $\mathrm{Tu}$ (EF-Tu; BBIF_1251) was amongst these proteins.

A total of $9.3 \%$ of the proteome have a (predicted) role in amino acid transport, biosynthesis, urea cycle, and metabolism of amino groups including 14 aminotransferases (Table 2) and 6.7\%

TABLE 1 | Relative abundance of functional categories according to clusters of orthologous groups of proteins (COGs) in the Bifidobacterium bifidum $\mathrm{S} 17$ proteome.

\begin{tabular}{ll} 
COG functional category & Abundance \\
\hline A: RNA processing and modification & $0.1 \%$ \\
C: Energy production and conversion & $3.0 \%$ \\
D: Cell cycle control, mitosis and meiosis & $1.1 \%$ \\
E: Amino acid transport and metabolism & $9.3 \%$ \\
F: Nucleotide transport and metabolism & $4.4 \%$ \\
G: Carbohydrate transport and metabolism & $6.7 \%$ \\
H: Coenzyme transport and metabolism & $4.9 \%$ \\
I: Lipid transport and metabolism & $2.7 \%$ \\
J: Translation & $11.9 \%$ \\
K: Transcription & $3.4 \%$ \\
L: Replication, recombination and repair & $4.4 \%$ \\
M: Cell wall/membrane biogenesis & $3.7 \%$ \\
N: Cell motility & $0.1 \%$ \\
O: Posttranslational modification, protein turnover, chaperones & $3.7 \%$ \\
P: Inorganic ion transport and metabolism & $3.0 \%$ \\
Q: Secondary metabolites biosynthesis, transport and catabolism & $1.9 \%$ \\
R: General function prediction only & $4.0 \%$ \\
S: Function unknown & $0.9 \%$ \\
T: Signal transduction mechanisms & $3.1 \%$ \\
U: Intracellular trafficking and secretion & $0.9 \%$ \\
V: Defense mechanisms & $2.7 \%$ \\
X: Transposons & $0.2 \%$ \\
-: Not in CoGs & $37.4 \%$
\end{tabular}


of all identified proteins are related to carbohydrate transport and metabolism. The major and characteristic metabolic pathway of bifidobacteria for fermentation of hexose sugars is the so-called fructose-6-phosphate or "bifid" shunt (Pokusaeva et al., 2011). We successfully detected a complete set of ten enzymes of the bifidus shunt (Figure 2A) including the key enzyme of the pathway xylulose-5-phosphate/fructose-6phosphate phosphoketolase (BBIF_0798). Moreover, 11 enzymes for a complete Embden-Meyerhof pathway were identified (Figure 2B).

Subcellular localization of all 1,148 proteins detected by LCMS/MS was predicted by PSORTb Version 3.0. Extracellular and cell wall proteins play critical roles in establishing and maintaining interactions between a microbe and its environment. Thus, proteins which, according to the PSORTB prediction, are located in the cell wall or extracellular were further analyzed for transmembrane helices and signal peptides by SignalP v4.1 and TMHMM Server v2.0 and searched or LPxTG cell wall anchor motifs using the LocateP database to confirm their cellular localization (Table 3). Interestingly, these analysis suggest a different localization for several of these proteins as predicted by PSORTB. For example, three of the 28 proteins (BBIF_0312, BBIF_0337, BBIF_1026) contained none of these domains/sequence motifs and are thus probably cytoplasmatic. Following, corrections the PSORTB correction of the proteins in Table 3, the proteome of B. bifidum S17 consists of 743 (64.7\%) cytoplasmic, 231 (21.1\%) membrane, $14(1.2 \%)$ cell wall, $3(0.3 \%)$ extracellular proteins and $146(12.7 \%)$ proteins with unknown cellular localization

TABLE 2 | Aminotransferases identified in the proteome of B. bifidum S17.

\begin{tabular}{|c|c|c|c|c|}
\hline Locus_tag & $\begin{array}{l}\text { Gene } \\
\text { name }\end{array}$ & Description & $\begin{array}{l}\text { Length } \\
\text { [aa] }^{\mathrm{a}}\end{array}$ & $\begin{array}{c}\text { MW } \\
{[k D a]^{b}}\end{array}$ \\
\hline BBIF_0278 & aspC1 & Aminotransferase & 401 & 42.8 \\
\hline BBIF_0311 & yhdR & Aspartate aminotransferase & 396 & 43.2 \\
\hline BBIF_0342 & bbif_0342 & $\begin{array}{l}\text { Multiple substrate } \\
\text { aminotransferase (MsaT) } \\
\text { containing domain of GntR family } \\
\text { (transcriptional regulator) }\end{array}$ & 509 & 55.6 \\
\hline BBIF_0469 & bbif_0469 & $\begin{array}{l}\text { Aspartate/tyrosine/aromatic } \\
\text { aminotransferase }\end{array}$ & 398 & 43.7 \\
\hline BBIF_0550 & hisC & $\begin{array}{l}\text { Histidinol-phosphate } \\
\text { aminotransferase }\end{array}$ & 391 & 43.2 \\
\hline BBIF_0701 & bbif_0701 & Aminotransferase & 370 & 40 \\
\hline BBIF_0741 & ilvE1 & $\begin{array}{l}\text { Branched-chain amino acid } \\
\text { aminotransferase }\end{array}$ & 262 & 29.1 \\
\hline BBIF_0863 & ilvE2 & $\begin{array}{l}\text { Branched-chain amino acid } \\
\text { aminotransferase }\end{array}$ & 375 & 41.5 \\
\hline BBIF_0870 & bbif_0870 & Aminotransferase & 522 & 58.1 \\
\hline BBIF_1100 & $\arg D$ & Acetylornithine aminotransferase & 429 & 45.4 \\
\hline BBIF_1175 & bbif_1175 & Aspartate aminotransferase & 394 & 43.5 \\
\hline BBIF_1428 & serC & Phosphoserine aminotransferase & 380 & 40.6 \\
\hline BBIF_1519 & bbif_1519 & $\begin{array}{l}\text { N-succinyldiaminopimelate } \\
\text { aminotransferase }\end{array}$ & 392 & 41.5 \\
\hline BBIF_1610 & aspC2 & Aspartate aminotransferase & 409 & 44.5 \\
\hline
\end{tabular}

aa, amino acids. ${ }^{b} k D a, k i l o$ Dalton.

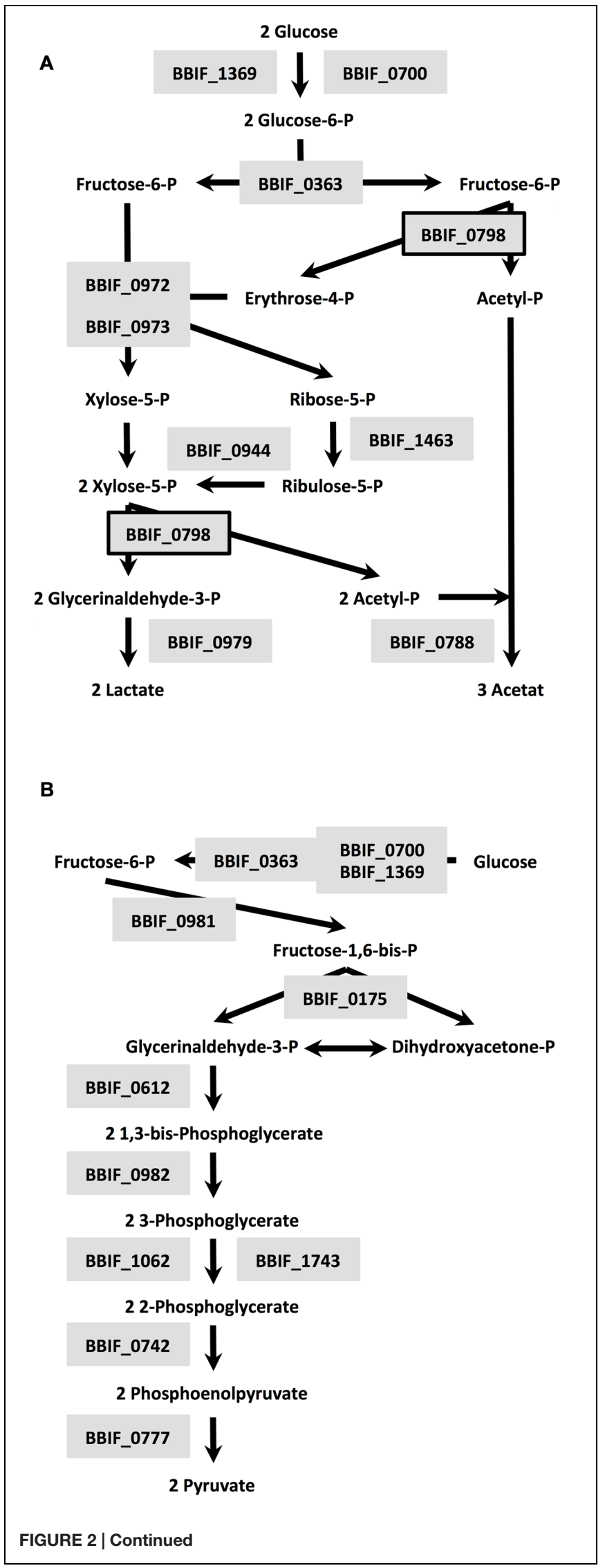




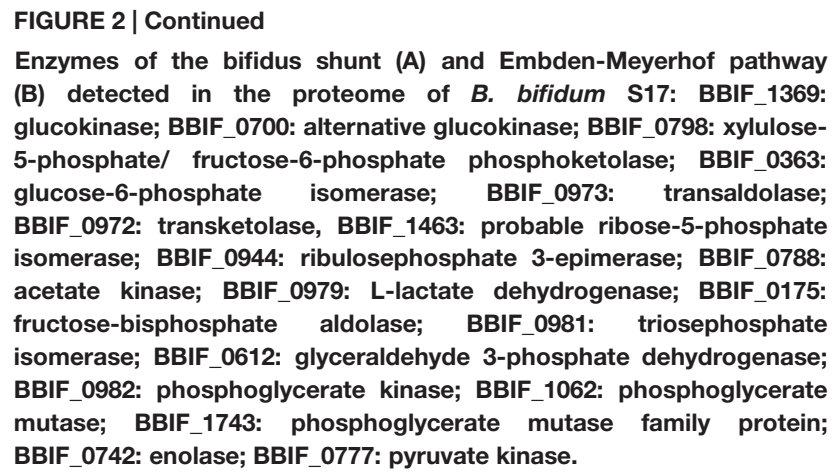

(Figure 3). A number of proteins of the B. bifidum S17 proteome with extracellular or cell wall localization have been associated with bacterial adhesion to host structures, colonization and/or immunomodulation. For example, five of the six pilin proteins for sortase-dependent pili including major pilins BBIF_0301 and BBIF_1761 and the minor pilins BBIF_0302; BBIF_1648, and BBIF_1761 were detected in the proteome. Further proteins identified in the proteome of B. bifidum S17 that may have a role in host colonization are the potential adhesin BBIF_0636 (BopA), the s subtilisin family peptidase BBIF_1681, and BBIF_1317 and BBIF_1734, two glycoside hydrolases of the fucosidase and sialidase family.

TABLE 3 | Proteins of the B. bifidum $\mathbf{S 1 7}$ proteome with predicted cell wall or extracellular localization.

\begin{tabular}{|c|c|c|c|c|c|c|}
\hline Locus_tag & Description & $\mathbf{S P}^{\mathrm{a}}$ & $\mathrm{TMH}^{\mathrm{b}}$ & Lipid anchor ${ }^{c}$ & CW anchor ${ }^{c}$ & $\begin{array}{l}\text { Final } \\
\text { prediction }^{d}\end{array}$ \\
\hline BBIF_0022 & Alpha-L-arabinofuranosidase & $1-36$ & 13-35, 1138-1160 & - & 1133-1138 (LSHTG) & $\mathrm{CW}$ \\
\hline BBIF_0285 & $\begin{array}{l}\text { Conserved hypothetical protein containing multiple sugar } \\
\text { recognition domains }\end{array}$ & $1-27$ & $5-27,1904-1926$ & - & 1899-1903 (ISKTG) & $\mathrm{CW}$ \\
\hline BBIF_0301 & $\begin{array}{l}\text { Conserved hypothetical protein containing von Willebrand } \\
\text { factor type A domain }\end{array}$ & $1-26$ & $5-27,1128-1150$ & - & 1118-1123 (LPMTG) & $\mathrm{CW}$ \\
\hline BBIF_0302 & Conserved hypothetical protein with Cna B-type domain & $1-29$ & $7-29,501-523$ & - & 496-501 (LPKTG) & CW \\
\hline BBIF_0507 & Beta-galactosidase BbgIII & $1-32$ & - & - & 1903-1907 (LSKTG) & $\mathrm{CW}$ \\
\hline BBIF_1317 & Alpha-L-fucosidase & $1-37$ & $13-35,1469-1491$ & - & $1466-1470$ (IAKTG) & $\mathrm{CW}$ \\
\hline BBIF_1382 & $\begin{array}{l}\text { Conserved hypothetical protein containing bacterial lg-like } \\
\text { domain (group 2) }\end{array}$ & $1-34$ & $13-35,1087-1106$ & - & 1079-1083 (LSATG) & CW \\
\hline BBIF_1461 & Beta-N-acetylglucosaminidase & $1-29$ & $1935-1954$ & - & 1926-1930 (ISKTG) & $\mathrm{CW}$ \\
\hline BBIF_1576 & Beta- $\mathrm{N}$-acetylglucosaminidase & $1-34$ & $12-34,1117-1139$ & - & $1112-1116$ (LSNTG) & CW \\
\hline BBIF_1648 & $\begin{array}{l}\text { Conserved hypothetical protein containing CnaB domain } \\
\text { and LPXTG-anchor }\end{array}$ & $1-29$ & $523-545$ & - & $518-523$ (LPLTG) & CW \\
\hline BBIF_1681 & Subtilisin family peptidase (lactocepin) & $1-28$ & $9-31,1325-1347$ & - & 1320-1324 (VAKTG) & $\mathrm{CW}$ \\
\hline BBIF_1734 & Sialidase & $1-35$ & $13-35,808-830$ & - & 803-807 (LSKTG) & $\mathrm{CW}$ \\
\hline BBIF_1761 & $\begin{array}{l}\text { Cell surface protein with gram positive anchor and Cna } \\
\text { protein B-type domains }\end{array}$ & $1-31$ & $7-29,505-527$ & - & 500-505 (LPGTG) & CW \\
\hline BBIF_1762 & Cell surface protein with LPXTG anchor & - & $2520-2542$ & & 2514-2519 (LPDTG) & CW \\
\hline BBIF_0048 & 1,4-beta-N-acetylmuramidase & $1-30$ & - & - & - & $E$ \\
\hline BBIF_0483 & Conserved protein with the pectin lyase fold domain & $1-30$ & - & - & - & $E$ \\
\hline BBIF_0522 & Conserved hypothetical protein with CHAP domain & $1-36$ & $9-31$ & - & - & $E$ \\
\hline BBIF_0158 & Trypsin-like serine protease & - & $203-225$ & - & - & M \\
\hline BBIF_0246 & Peptidylprolylisomerase, FKBP-type & $1-36$ & $13-32$ & 24-30 (VTLAACG) & - & M \\
\hline BBIF_0313 & Hypothetical protein BBIF_0313 & - & $236-258$ & - & - & M \\
\hline BBIF_0592 & Peptide/nickel transport system, substrate-binding protein & $1-28$ & $7-26$ & 18-24 (ASLTACG) & - & M \\
\hline BBIF_0636 & $\begin{array}{l}\text { Peptide/nickel transport system, extracellular } \\
\text { solute-binding protein (BopA) }\end{array}$ & $1-34$ & - & $21-27$ (LALGACG) & - & M \\
\hline BBIF_1309 & Peptide/nickel transport system, substrate-binding protein & - & $55-77$ & - & - & M \\
\hline BBIF_1426 & Conserved hypothetical protein with NIpC/P60 domain & $1-25$ & $16-38$ & - & - & M \\
\hline BBIF_1605 & ABC transporter solute-binding protein & - & $13-35$ & - & - & M \\
\hline BBIF_0312 & Conserved hypothetical protein & - & - & - & - & $\mathrm{CP}$ \\
\hline BBIF_0337 & Hsp20-family heat shock chaperone & - & - & - & - & $\mathrm{CP}$ \\
\hline BBIF_1026 & DNA polymerase III, delta subunit & - & - & - & - & $\mathrm{CP}$ \\
\hline
\end{tabular}

a SP, signal peptide predicted using SignalP V4.1.

b TMH, transmembrane helices, predicted usingTMHMM Server v2.0.

'Lipid anchor and cell wall (CW) anchor motifs obtained from the LocateP database (http://www.cmbi.ru.nl/locatep-db/cgi-bin/locatepdb.py).

dfinal prediction for cellular localization cell wall (CW), extracellular (E), membrane (M), orcytoplasma (Cp). 


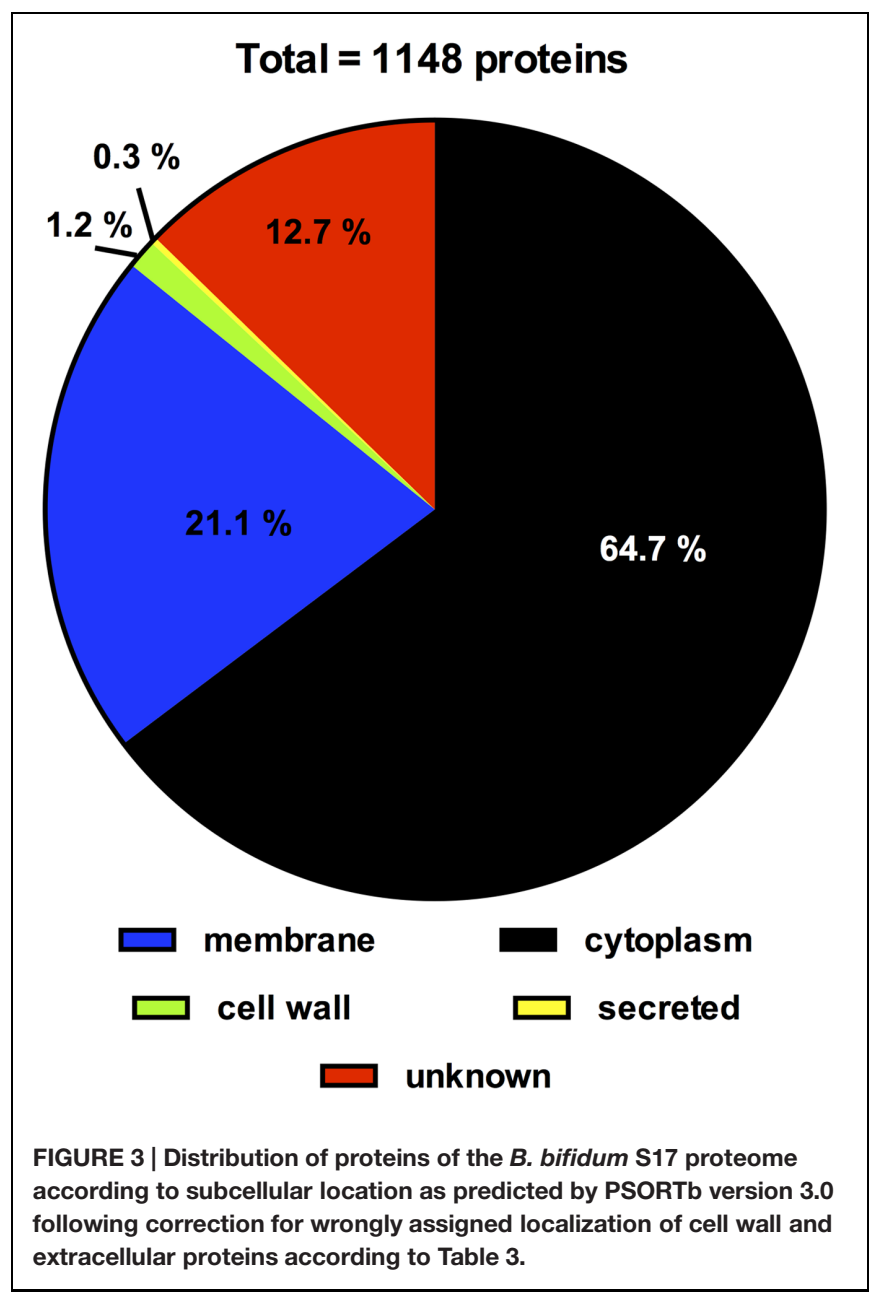

\section{DISCUSSION}

Bifidobacterium bifidum strains belong to the infant-type bifidobacteria and some of these strains possess interesting properties related to host-health (Turroni et al., 2014a). In an attempt to compile the first proteomic reference profile for the species, B. bifidum, we determined the complete proteome of B. bifidum S17 grown in MRS, i.e., the standard medium for routine culture of bifidobacteria, by LC-MS/MS. In total 1,148 proteins were detected, i.e., a coverage $64.4 \%$ of all predicted proteins of B. bifidum S17. This is comparable to the coverage obtained by proteome analysis of two L. rhamnosus strains (Savijoki et al., 2011). However, it may still be an underestimation of the complete proteome of B. bifidum S17 expressed under the conditions tested. The experimental approach utilized the bacterial pellet of cultures grown in MRS. Thus, most proteins that are secreted into the cell culture supernatant are probably not captured. In line with the proteomes of other bacteria (Yuan et al., 2006; Liu et al., 2012), proteins with a function in DNA replication, recombination and repair, RNA processing, transcription, and translation to were highly prevalent in the proteome of B. bifidum S17. One of these proteins is elongation factors Tu (EF-Tu; BBIF_1251), which was previously shown to posses moonlighting function as an adhesin that mediates binding of B. longum NCC2705 and Lactobacillus sp. mucus, IECs, and/or ECM components of the host (Granato et al., 2004; Ramiah et al., 2008; Dhanani and Bagchi, 2013; Wei et al., 2014).

Detection of large numbers of enzymes of the bifidus shunt, glycolysis, and amino acid metabolism in the proteome of $B$. bifidum S17 confirms previous findings on the proteome of B. longum NCC2705 (Yuan et al., 2006). B. bifidum S17 was grown in MRS and B. longum NCC2705 in modified Garches medium for proteome analysis. Both media contain high levels of complex components such as yeast extract, peptone and/or beef extract and glucose as additional carbon source (De Man et al., 1960; Krzewinski et al., 1997). This may explain the high numbers of enzymes detected in the two proteomes involved in the uptake, degradation and fermentation of these substrates. Similar to EF-Tu, one of the proteins of the Embden-Meyerhof pathway is a cytoplasmic enzyme that has a moonlighting function. Enolase (BBIF_0742) was shown to mediate adhesion of B. bifidum and other bifidobacteria to IECs and components of the ECM (Candela et al., 2009; Wei et al., 2014). An interesting finding is the detection of 14 aminotransferases in the proteome (Table 2). Transamination reactions of amino acid converting pathways have recently attracted attention because they are the first step for the synthesis of important aroma compounds (Christensen et al., 1999) which may affect flavor of probiotic preparations.

A number of proteins of the B. bifidum S17 proteome contain domains and signal sequences for extracellular or cell wall location. Some of these proteins have been associated with bacterial adhesion to host structures, colonization and/or immunomodulation. Five of the six pilin proteins for sortasedependent pili were identified in the proteome including major pilins BBIF_0301 and BBIF_1761 and the minor pilins BBIF_0302; BBIF_1648, and BBIF_1761. This suggests that at least two of the three sortase-dependent pili encoded on the genome of B. bifidum S17 are expressed under laboratory conditions. This is in line with the observation that sortasedependent pili gene clusters are expressed by B. bifidum PRL2010 both in vitro and in the mouse GIT (Turroni et al., 2013) and pilus-like structures are detectable by atomic force microscopy in the same strain in vitro (Foroni et al., 2011). Expression of pili may have an important function in interaction of B. bifidum strains with the host. Heterologous expression of one of these gene clusters in L. lactis led to increased adhesion to ECM proteins and cultured IECs (Turroni et al., 2013). Moreover, the L. lactis strain expressing these pili elicits altered cytokine profiles in the murine gastrointestinal mucosa compared to the control. Another protein with potential role in adhesion to host structures is BBIF_0636. This protein is an extracellular solute-binding protein of a peptide/nickel transport system, which was also termed bifidobacterial outer protein A (BopA). Previous data obtained by us and others suggests that BopA mediates binding to cultured human IECs (Guglielmetti et al., 2008; Gleinser et al., 2012). However, these findings have been challenged recently (Kainulainen et al., 2013). 
Three further proteins of the B. bifidum S17 proteome with a potential role in interaction with the host are BBIF_1317, BBIF_1681, and BBIF_1734. BBIF_1681 is a peptidase of the subtilisin family. The subtilisin family peptidase lactocepin of L. casei was shown to degrade pro-inflammatory cytokines contributing to the immunomodulatory effect of this probiotic bacterium (von Schillde et al., 2012). BBIF_1317 and BBIF_1734 are a glycoside hydrolases of the fucosidase and sialidase family, respectively. Sialidases are known as important virulence factors of bacterial pathogens that mediate attachment and degradation of host-derived mucus and were also shown to be involved in host colonization by commensal bacteria (Lewis and Lewis, 2012). Genes for mucin degradation pathways including sialidases are conserved amongst B. bifidum strains and most $B$. bifidum strains are able to grow on mucin as sole carbon source (Turroni et al., 2010). Similarly, fucosidases are involved in degradation of host-derived glucans such as human milk oligosaccharides and mucus (Turroni et al., 2010). Utilization of host-derived glucans is considered as nutritional adaptation to the intestinal tract of the (human) host (Turroni et al., 2010; Bottacini et al., 2014; Grimm et al., 2014). Thus, detection of proteins for degradation of host derived glycans under in vitro is somewhat surprising since these substrates are no present in standard growth medium, i.e., MRS. However, LC-MS/MS is a highly sensitive method that allows detection of even small amounts of a given protein (Otto et al., 2014). Moreover, pilin proteins that are only required in vivo were also present in the proteome of $B$. bifidum S17 and expression of pili genes and proteins in vitro has been observed previously (Foroni et al., 2011; Westermann et al., 2012). This suggests that regulation of host-interacting proteins may not strictly regulated in bifidobacteria.

In summary a total of 1,148 proteins of the predicted proteome were detected including important metabolic pathways, proteins

\section{REFERENCES}

Albrethsen, J., Knol, J. C., Piersma, S. R., Pham, T. V., de Wit, M., Mongera, S., et al. (2010). Subnuclear proteomics in colorectal cancer: identification of proteins enriched in the nuclear matrix fraction and regulation in adenoma to carcinoma progression. Mol. Cell. Proteomics 9, 988-1005. doi: 10.1074/mcp.M900546MCP200

Altschul, S. F., Madden, T. L., Schäffer, A. A., Zhang, J., Zhang, Z., Miller, W., et al. (1997). Gapped BLAST and PSI-BLAST: a new generation of protein database search programs. Nucleic Acids Res. 25, 3389-3402. doi: 10.1093/nar/25.17.3389

Bottacini, F., Ventura, M., van Sinderen, D., and O'Connell Motherway, M. (2014). Diversity, ecology and intestinal function of bifidobacteria. Microb. Cell Fact. 13(Suppl. 1), S4. doi: 10.1186/1475-2859-13-S1-S4

Burtnick, M. N., Brett, P. J., and DeShazer, D. (2014). Proteomic analysis of the Burkholderia pseudomallei type II secretome reveals hydrolytic enzymes, novel proteins, and the deubiquitinase TssM. Infect. Immun. 82, 3214-3226. doi: 10.1128/IAI.01739-14

Candela, M., Biagi, E., Centanni, M., Turroni, S., Vici, M., Musiani, F., et al. (2009). Bifidobacterial enolase, a cell surface receptor for human plasminogen involved in the interaction with the host. Microbiology 155, 3294-3303. doi: 10.1099/mic.0.028795-0

Christensen, J. E., Dudley, E. G., Pederson, J. A., and Steele, J. L. (1999). Peptidases and amino acid catabolism in lactic acid bacteria. Antonie Van Leeuwenhoek 76, 217-246. doi: 10.1023/A:100200191 9720 known or suspected to be involved in adhesion and colonization as well as a large number of (previously) hypothetical proteins. This represents the first complete proteome analysis of the species $B$. bifidum and confirms previous findings on the proteome level.

\section{AUTHOR CONTRIBUTIONS}

JY, CR, and XW designed research; XW, DZ, HL, and WL performed research; XW, SW, BL, and XZ contributed new reagents or analytic tools; XW, JL, and CR analyzed data; XW, $\mathrm{CR}$, and YS wrote the paper.

\section{FUNDING}

This work was supported by a grant from the National Natural Science Foundation of China (31370093) to JY and (81400592) to XW, Mega-projects of Science and Technology Research of China (Grant 2011ZX10004-001), the German Academic Exchange Service/Federal Ministry of Education and Research to CR (Grant D/09/04778), and a grant from the National High Technology Research and Development Program of China (863 Program; grant no. SS2014AA022210).

\section{SUPPLEMENTARY MATERIAL}

The Supplementary Material for this article can be found online at: http://journal.frontiersin.org/article/10.3389/fmicb. 2016.00097

TABLE S1 | Characteristics of the 1,148 identified proteins.

De Man, J. C., Rogosa, M., and Sharpe, M. E. (1960). A medium for the cultivation of lactobacilli. J. Appl. Microbiol. 23, 130-135. doi: 10.1111/j.13652672.1960.tb00188.x

Dhanani, A. S., and Bagchi, T. (2013). The expression of adhesin EF-Tu in response to mucin and its role in Lactobacillus adhesion and competitive inhibition of enteropathogens to mucin. J. Appl. Microbiol. 115, 546-554. doi: 10.1111/jam.12249

Fanning, S., Hall, L. J., Cronin, M., Zomer, A., Macsharry, J., Goulding, D., et al. (2012). Bifidobacterial surface-exopolysaccharide facilitates commensal-host interaction through immune modulation and pathogen protection. Proc. Natl. Acad. Sci. U.S.A. 109, 2108-2113. doi: 10.1073/pnas.1115621109

Foroni, E., Serafini, F., Amidani, D., Turroni, F., He, F., Bottacini, F., et al. (2011). Genetic analysis and morphological identification of pilus-like structures in members of the genus Bifidobacterium. Microb. Cell Fact. 10(Suppl. 1), S16. doi: 10.1186/1475-2859-10-S1-S16

Gareau, M. G., Sherman, P. M., and Walker, W. A. (2010). Probiotics and the gut microbiota in intestinal health and disease. Nat. Rev. Gastroenterol. Hepatol. 7, 503-514. doi: 10.1038/nrgastro.2010.117

Gleinser, M., Grimm, V., Zhurina, D., Yuan, J., and Riedel, C. U. (2012). Improved adhesive properties of recombinant bifidobacteria expressing the Bifidobacterium bifidum-specific lipoprotein BopA. Microb. Cell Fact. 11, 80. doi: 10.1186/1475-2859-11-80

Granato, D., Bergonzelli, G. E., Pridmore, R. D., Marvin, L., Rouvet, M., and Corthésy-Theulaz, I. E. (2004). Cell surface-associated elongation factor $\mathrm{Tu}$ mediates the attachment of Lactobacillus johnsonii NCC533 (La1) to 
human intestinal cells and mucins. Infect. Immun. 72, 2160-2169. doi: 10.1128/IAI.72.4.2160-2169.2004

Grimm, V., Radulovic, K., and Riedel, C. U. (2015). Colonization of C57BL/6 mice by a potential probiotic Bifidobacterium bifidum strain under germ-free and specific pathogen-free conditions and during experimental colitis. PLOS ONE 10:e0139935. doi: 10.1371/journal.pone.0139935

Grimm, V., Westermann, C., and Riedel, C. U. (2014). Bifidobacteria-host interactions-an update on colonisation factors. Biomed Res. Int. 2014, 960826. doi: $10.1155 / 2014 / 960826$

Guglielmetti, S., Tamagnini, I., Mora, D., Minuzzo, M., Scarafoni, A., Arioli, S., et al. (2008). Implication of an outer surface lipoprotein in adhesion of Bifidobacterium bifidum to Caco-2 cells. Appl. Environ. Microbiol. 74, 46954702. doi: 10.1128/AEM.00124-08

Guillaume, E., Berger, B., Affolter, M., and Kussmann, M. (2009). Label-free quantitative proteomics of two Bifidobacterium longum strains. J. Proteomics 72, 771-784. doi: 10.1016/j.jprot.2009.03.004

Kainulainen, V., Reunanen, J., Hiippala, K., Guglielmetti, S., Vesterlund, S., Palva, A., et al. (2013). BopA has no major role in the adhesion of Bifidobacterium bifidum to intestinal epithelial cells, extracellular matrix proteins and mucus. Appl. Environ. Microbiol. 79, 6989-6997. doi: 10.1128/AEM.01993-13

Kim, J.-H., An, H. J., Garrido, D., German, J. B., Lebrilla, C. B., and Mills, D. A. (2013). Proteomic analysis of Bifidobacterium longum subsp. infantis reveals the metabolic insight on consumption of prebiotics and host glycans. PLoS ONE 8:e57535. doi: 10.1371/journal.pone.0057535

Krzewinski, F., Brassart, C., Gavini, F., and Bouquelet, S. (1997). Glucose and galactose transport in Bifidobacterium bifidum DSM 20082. Curr. Microbiol. 35, 175-179. doi: 10.1007/s002849900234

Leahy, S. C., Higgins, D. G., Fitzgerald, G. F., and van Sinderen, D. (2005). Getting better with bifidobacteria. J. Appl. Microbiol. 98, 1303-1315. doi: 10.1111/j.1365-2672.2005.02600.x

Lewis, A. L., and Lewis, W. G. (2012). Host sialoglycans and bacterial sialidases: a mucosal perspective. Cell. Microbiol. 14, 1174-1182. doi: 10.1111/j.14625822.2012.01807.x

Liu, D., Wang, S., Xu, B., Guo, Y., Zhao, J., Liu, W., et al. (2011). Proteomics analysis of Bifidobacterium longum NCC2705 growing on glucose, fructose, mannose, xylose, ribose, and galactose. Proteomics 11, 2628-2638. doi: $10.1002 /$ pmic. 201100035

Liu, S., Ren, F., Zhao, L., Jiang, L., Hao, Y., Jin, J., et al. (2015). Starch and starch hydrolysates are favorable carbon sources for Bifidobacteria in the human gut. BMC Microbiol. 15:54. doi: 10.1186/s12866-015-0362-3

Liu, Y.-C., Lin, I.-H., Chung, W.-J., Hu, W. S., Ng, W. V., Lu, C.-Y., et al. (2012). Proteomics characterization of cytoplasmic and lipid-associated membrane proteins of human pathogen Mycoplasma fermentans M64. PLoS ONE 7:e35304. doi: 10.1371/journal.pone.0035304

O'Connell Motherway, M., Zomer, A., Leahy, S. C., Reunanen, J., Bottacini, F., Claesson, M. J., et al. (2011). Functional genome analysis of Bifidobacterium breve UCC2003 reveals type IVb tight adherence (Tad) pili as an essential and conserved host-colonization factor. Proc. Natl. Acad. Sci. U.S.A. 108, 11217-11222. doi: 10.1073/pnas.1105380108

Otto, A., Becher, D., and Schmidt, F. (2014). Quantitative proteomics in the field of microbiology. Proteomics 14, 547-565. doi: 10.1002/pmic.201300403

Petersen, T. N., Brunak, S., von Heijne, G., and Nielsen, H. (2011). SignalP 4.0: discriminating signal peptides from transmembrane regions. Nat. Methods 8 , 785-786. doi: 10.1038/nmeth.1701

Philippe, D., Heupel, E., Blum-Sperisen, S., and Riedel, C. U. (2011). Treatment with Bifidobacterium bifidum 17 partially protects mice from Th1-driven inflammation in a chemically induced model of colitis. Int. J. Food Microbiol. 149, 45-49. doi: 10.1016/j.ijfoodmicro.2010.12.020

Pokusaeva, K., Fitzgerald, G. F., and van Sinderen, D. (2011). Carbohydrate metabolism in Bifidobacteria. Genes Nutr. 6, 285-306. doi: 10.1007/s12263-0100206-6

Preising, J., Philippe, D., Gleinser, M., Wei, H., Blum, S., Eikmanns, B. J., et al. (2010). Selection of bifidobacteria based on adhesion and anti-inflammatory capacity in vitro for amelioration of murine colitis. Appl. Environ. Microbiol. 76, 3048-3051. doi: 10.1128/AEM.03127-09

Ramiah, K., van Reenen, C. A., and Dicks, L. M. T. (2008). Surface-bound proteins of Lactobacillus plantarum 423 that contribute to adhesion of Caco-2 cells and their role in competitive exclusion and displacement of
Clostridium sporogenes and Enterococcus faecalis. Res. Microbiol. 159, 470-475. doi: 10.1016/j.resmic.2008.06.002

Riedel, C. U., Foata, F., Goldstein, D. R., Blum, S., and Eikmanns, B. J. (2006a). Interaction of bifidobacteria with Caco-2 cells-adhesion and impact on expression profiles. Int. J. Food Microbiol. 110, 62-68. doi: 10.1016/j.ijfoodmicro.2006.01.040

Riedel, C. U., Foata, F., Philippe, D., Adolfsson, O., Eikmanns, B. J., and Blum, S. (2006b). Anti-inflammatory effects of bifidobacteria by inhibition of LPSinduced NF-kappaB activation. World J. Gastroenterol. 12, 3729-3735.

Sánchez, B., Champomier-Vergès, M.-C., Collado, M., del, C., Anglade, P., Baraige, F., et al. (2007). Low-pH adaptation and the acid tolerance response of Bifidobacterium longum biotype longum. Appl. Environ. Microbiol. 73, 64506459. doi: 10.1128/AEM.00886-07

Savijoki, K., Lietzeìn, N., Kankainen, M., Alatossava, T., Koskenniemi, K. Varmanen, P., et al. (2011). Comparative proteome cataloging of Lactobacillus rhamnosus strains GG and Lc705. J. Proteome Res. 10, 3460-3473. doi: $10.1021 /$ pr2000896

Sonnhammer, E. L., von Heijne, G., and Krogh, A. (1998). A hidden Markov model for predicting transmembrane helices in protein sequences. Proc. Int. Conf. Intell. Syst. Mol. Biol. 6, 175-182.

Tatusov, R. L., Galperin, M. Y., Natale, D. A., and Koonin, E. V. (2000). The COG database: a tool for genome-scale analysis of protein functions and evolution. Nucleic Acids Res. 28, 33-36. doi: 10.1093/nar/28.1.33

Turroni, F., Bottacini, F., Foroni, E., Mulder, I., Kim, J.-H., Zomer, A., et al. (2010). Genome analysis of Bifidobacterium bifidum PRL2010 reveals metabolic pathways for host-derived glycan foraging. Proc. Natl. Acad. Sci. U.S.A. 107, 19514-19519. doi: 10.1073/pnas.1011100107

Turroni, F., Duranti, S., Bottacini, F., Guglielmetti, S., Van Sinderen, D., and Ventura, M. (2014a). Bifidobacterium bifidum as an example of a specialized human gut commensal. Front. Microbiol. 5:437. doi: 10.3389/fmicb.2014.00437

Turroni, F., Serafini, F., Mangifesta, M., Arioli, S., Mora, D., van Sinderen, D., et al. (2014b). Expression of sortase-dependent pili of Bifidobacterium bifidum PRL2010 in response to environmental gut conditions. FEMS Microbiol. Lett. 357, 23-33. doi: 10.1111/1574-6968.12509

Turroni, F., Serafini, F., Foroni, E., Duranti, S., O'Connell Motherway, M., Taverniti, V., et al. (2013). Role of sortase-dependent pili of Bifidobacterium bifidum PRL2010 in modulating bacterium-host interactions. Proc. Natl. Acad. Sci. U.S.A. 110, 11151-11156. doi: 10.1073/pnas.1303897110

Ventura, M., O’Flaherty, S., Claesson, M. J., Turroni, F., Klaenhammer, T. R., van Sinderen, D., et al. (2009). Genome-scale analyses of health-promoting bacteria: probiogenomics. Nat. Rev. Microbiol. 7, 61-71. doi: 10.1038/nrmicro2047

von Schillde, M.-A., Hörmannsperger, G., Weiher, M., Alpert, C.-A., Hahne, H., Bäuerl, C., et al. (2012). Lactocepin secreted by Lactobacillus exerts antiinflammatory effects by selectively degrading proinflammatory chemokines. Cell Host Microbe 11, 387-396. doi: 10.1016/j.chom.2012.02.006

Wei, X., Yan, X., Chen, X., Yang, Z., Li, H., Zou, D., et al. (2014). Proteomic analysis of the interaction of Bifidobacterium longum NCC2705 with the intestine cells Caco-2 and identification of plasminogen receptors. J. Proteomics 108, 89-98. doi: 10.1016/j.jprot.2014.04.038

Westermann, C., Zhurina, D. S., Baur, A., Shang, W., Yuan, J., and Riedel, C. U. (2012). Exploring the genome sequence of Bifidobacterium bifidum S17 for potential players in host-microbe interactions. Symbiosis 58, 191-200. doi: 10.1007/s13199-012-0205-z

Wickramasekara, S., Neilson, J., Patel, N., Breci, L., Hilderbrand, A., Maier, R. M., et al. (2011). Proteomics analyses of the opportunistic pathogen Burkholderia vietnamiensis using protein fractionations and mass spectrometry. J. Biomed. Biotechnol. 2011, 701928. doi: 10.1155/2011/701928

Xiao, M., Xu, P., Zhao, J., Wang, Z., Zuo, F., Zhang, J., et al. (2011). Oxidative stress-related responses of Bifidobacterium longum subsp. longum BBMN68 at the proteomic level after exposure to oxygen. Microbiology 157, 1573-1588. doi: 10.1099/mic.0.044297-0

Yu, N. Y., Wagner, J. R., Laird, M. R., Melli, G., Rey, S., Lo, R., et al. (2010). PSORTb 3.0: improved protein subcellular localization prediction with refined localization subcategories and predictive capabilities for all prokaryotes. Bioinformatics 26, 1608-1615. doi: 10.1093/bioinformatics/btq249

Yuan, J., Wang, B., Sun, Z., Bo, X., Yuan, X., He, X., et al. (2008). Analysis of host-inducing proteome changes in Bifidobacterium longum NCC2705 grown in vivo. J. Proteome Res. 7, 375-385. doi: 10.1021/pr07 04940 
Yuan, J., Zhu, L., Liu, X., Li, T., Zhang, Y., Ying, T., et al. (2006). A proteome reference map and proteomic analysis of Bifidobacterium longum NCC2705. Mol. Cell. Proteomics 5, 1105-1118. doi: 10.1074/mcp.M500410MCP200

Zhou, M., Boekhorst, J., Francke, C., and Siezen, R. J. (2008). LocateP: genomescale subcellular-location predictor for bacterial proteins. BMC Bioinformatics 9:173. doi: 10.1186/1471-2105-9-173

Zhurina, D., Zomer, A., Gleinser, M., Brancaccio, V. F., Auchter, M., Waidmann, M. S., et al. (2011). Complete genome sequence of Bifidobacterium bifidum S17. J. Bacteriol. 193, 301-302. doi: 10.1128/JB. 01180-10
Conflict of Interest Statement: The authors declare that the research was conducted in the absence of any commercial or financial relationships that could be construed as a potential conflict of interest.

Copyright (C) 2016 Wei, Wang, Zhao, Wang, Li, Lin, Lu, Zhurina, Li, Riedel, Sun and Yuan. This is an open-access article distributed under the terms of the Creative Commons Attribution License (CC BY). The use, distribution or reproduction in other forums is permitted, provided the original author(s) or licensor are credited and that the original publication in this journal is cited, in accordance with accepted academic practice. No use, distribution or reproduction is permitted which does not comply with these terms. 